\title{
Constraints and degrees of freedom in Lorentz-violating field theories
}

\author{
Michael D. Seifert ${ }^{*}$ \\ Department of Physics, Astronomy, and Geophysics, Connecticut College, \\ 270 Mohegan Ave., New London, CT 06320, USA
}

(Received 10 December 2018; published 7 February 2019)

\begin{abstract}
Many current models which "violate Lorentz symmetry" do so via a vector or tensor field which takes on a vacuum expectation value, thereby spontaneously breaking the underlying Lorentz symmetry of the Lagrangian. To obtain a tensor field with this behavior, one can posit a smooth potential for this field, in which case it would be expected to lie near the minimum of its potential. Alternately, one can enforce a nonzero tensor value via a Lagrange multiplier. The present work explores the relationship between these two types of theories in the case of vector models. In particular, the naïve expectation that a Lagrange multiplier "kills off" 1 degree of freedom via its constraint does not necessarily hold for vector models that already contain primary constraints. It is shown that a Lagrange multiplier can only reduce the degrees of freedom of a model if the field-space function defining the vacuum manifold commutes with the primary constraints.
\end{abstract}

DOI: 10.1103/PhysRevD.99.045003

\section{INTRODUCTION}

Many classical field theories are constructed in such a way that the "most natural" solutions to the equations of motion involve a nonzero field value. This paradigm, where an underlying symmetry of the Lagrangian or Hamiltonian is spontaneously broken by the solutions of the equations of motion, has proven to be both compelling and fruitful over the years. In the context of particle physics, the bestknown example is the Higgs field [1-4]; in the context of condensed-matter physics, this paradigm underlies the modern theory of phase transitions, most notably the Ginzburg-Landau theory of superconductivity [5]. Many nonlinear sigma models can also be thought of in this way, if one view the model's target manifold as being embedded in some higher-dimensional space in which the fields are forced to a nonzero value. Such models have been used in the study of both particle physics [6,7] and in ferromagnetism [8].

In more recent years, this paradigm has also been used to study possible observational signatures of Lorentz symmetry violation. Such models include a new vector or tensor field and have equations of motion that are satisfied when the metric is flat and the new tensor field is constant but nonzero. In this sense, Lorentz symmetry is spontaneously broken in these theories, as the tensor field takes on a vacuum expectation value that has nontrivial

\footnotetext{
*mseifer1@ conncoll.edu
}

Published by the American Physical Society under the terms of the Creative Commons Attribution 4.0 International license. Further distribution of this work must maintain attribution to the author(s) and the published article's title, journal citation, and DOI. Funded by SCOAP. transformation properties under the Lorentz group. The vacuum state of such a model is often said to be "Lorentzviolating", though it would be more accurate to say that Lorentz symmetry is spontaneously broken in the model. Examples of such models can be found in [9-14], as well as a particularly early example in [15].

In general, all of these models (Lorentz-violating or otherwise) have the property that the fields, collectively denoted by $\psi^{\alpha}$, will satisfy at least one equation of the form $f\left(\psi^{\alpha}\right)=0$ in the "vacuum", appropriately defined. Here, $f$ is a real-valued function of the fields; thus, if the fields $\psi^{\alpha}$ are specified by $N$ real numbers, the equation $f\left(\psi^{\alpha}\right)=0$ will generically specify an $(N-1)$-dimensional hypersurface in field space. I will therefore refer to this function as the vacuum manifold function.

Two broad classes of models in which some fields have nontrivial background values can readily be conceived of. In one class, the constraint $f\left(\psi^{\alpha}\right)=0$ is enforced exactly via the introduction of a Lagrange multiplier $\lambda$ in the Lagrange density,

$$
\mathcal{L}_{\mathrm{LM}}=\left(\nabla \psi^{\alpha}\right)\left(\nabla \psi^{\alpha}\right)+\lambda f\left(\psi^{\alpha}\right)
$$

where we have denoted the kinetic terms for the fields $\psi^{\alpha}$ schematically. I will call such models Lagrange-multiplier (LM) models.

In the other class, the fields $\psi^{\alpha}$ are assigned a potential energy that is minimized when the field is nonzero. In particular, if we define a potential $V\left(\psi^{\alpha}\right) \propto f^{2}\left(\psi^{\alpha}\right)$ and write down a Lagrange density,

$$
\mathcal{L}_{P}=\left(\nabla \psi^{\alpha}\right)\left(\nabla \psi^{\alpha}\right)-V\left(\psi^{\alpha}\right),
$$


then the lowest-energy state of the theory would be expected to occur when $f\left(\psi^{\alpha}\right)=0$ and $\nabla \psi^{\alpha}=0$. I will call such models potential models.

A natural question then arises: for a given collection of fields $\psi^{\alpha}$ and a given kinetic term $\left(\nabla \psi^{\alpha}\right)\left(\nabla \psi^{\alpha}\right)$, what is the relationship between the models (1) and (2)? In particular, one might expect the following statement to be true:

Conjecture: In a potential model such as (2), the fields can take on any value in the $N$-dimensional field space. By contrast, in a Lagrange-multiplier model, the fields are constrained to an $(N-1)$-dimensional subspace of field space. Thus, the number of degrees of freedom (d.o.f.) of a Lagrange-multiplier model (1) should be one fewer than the d.o.f. of the corresponding potential model (2).

The main purpose of this article is to show that this naïve conjecture is not true in general; in particular, if $\psi^{\alpha}$ includes a spacetime vector or tensor field, it may be false. In such models, the fields may need to satisfy certain constraints due to the structure of the kinetic terms; adding a new "constraint" to such theories, in the form of a Lagrange multiplier, does not automatically reduce the number of d.o.f. of the theory.

To demonstrate this, I will analyze the d.o.f. of two types of symmetry-breaking models. After a brief description of Dirac-Bergmann analysis in Sec. II, I will first analyze a multiplet of Lorentz scalar fields with an internal symmetry, followed by a vector field (Secs. III and IV respectively.) For the vector fields, the analysis will depend on the structure of the kinetic term chosen, and so three distinct sub-cases will need to be treated. In each case, I will examine a model where the fields are assigned a potential energy, and one where the fields are directly constrained via a Lagrange multiplier.

The bulk of the explicit analysis in this work will be done in the context of a fixed, flat background spacetime; however, I will briefly discuss these models in the context of a dynamical curved spacetime in Sec. V. In that section, I will also discuss the implications of these results for the broader relationship between these two classes of models. More general tensor fields will be examined in a forthcoming work [16].

Throughout this work, I will use units in which $\hbar=c=1$; the metric signature will be $(-+++)$. Roman indices $a, b, c, \ldots$ will be used to denote spacetime tensor indices; $i, j, k, \ldots$ will be used to denote spatial indices, where necessary. Greek indices $\alpha, \beta, \gamma, \ldots$ will be used exclusively to denote indices in field space. All expressions involving repeated indices (either tensor indices or field space indices) can be assumed to obey the Einstein summation convention.

\section{DIRAC-BERGMANN ANALYSIS}

Our primary tool for finding the number of degrees of each model will be Dirac-Bergmann constraint analysis [17]; my methods and nomenclature below will draw heavily from the later work of Isenberg and Nester [18]. I will briefly summarize the method here, and then illustrate it in more detail via the example theories described in Sec. III.

The method of Dirac-Bergmann analysis involves the construction of a Hamiltonian which generates the timeevolution of the system. In the process of this construction, one may need to introduce constraints among various variables, thereby reducing the number of d.o.f. of the system. One may also discover that the evolution of certain field combinations is undetermined by the equations of motion (e.g., gauge d.o.f.). These combinations of fields, which we will collectively call "gauges", must be interpreted as unphysical, again reducing the number of physical d.o.f. of the model.

If, as is usual, we count a field d.o.f. as a pair of realvalued functions (e.g., a field value and its conjugate momentum) that can be freely specified on an initial data surface, then the number of d.o.f. $N_{\text {dof }}$ can be inferred quite simply once the above analysis is complete,

$$
\begin{aligned}
N_{\text {dof }}= & \frac{1}{2}\left[\left(\begin{array}{c}
\text { no.of } \\
\text { fields }
\end{array}\right)+\left(\begin{array}{c}
\text { no.of } \\
\text { momenta }
\end{array}\right)-\left(\begin{array}{c}
\text { no.of } \\
\text { constraints }
\end{array}\right)\right. \\
& \left.-\left(\begin{array}{c}
\text { no.of } \\
\text { gauges }
\end{array}\right)\right] .
\end{aligned}
$$

In general, of course, the number of fields and the number of conjugate momenta will be the same. Moreover, in the particular field theories I will be considering in this work, I will not find any "gauges", so the last term in (3) will vanish. Thus, for my purposes, the above equation reduces to

$$
N_{\text {dof }}=\left(\begin{array}{c}
\text { no.of } \\
\text { fields }
\end{array}\right)-\frac{1}{2}\left(\begin{array}{c}
\text { no.of } \\
\text { constraints }
\end{array}\right) .
$$

\section{SCALAR MULTIPLET FIELDS}

The first case we will consider is a multiplet of $N$ Lorentz scalars: $\psi^{\alpha}=\phi^{\alpha}$, with $\alpha=1,2,3, \ldots, N$. We wish to construct a model where these scalars "naturally" take on values in some $(N-1)$-dimensional hypersurface, defined by $f\left(\phi^{\alpha}\right)=0$ (with $f$ a real-valued function). The "potential model" for this field will be derived from the Lagrange density

$$
\begin{aligned}
\mathcal{L} & =-\frac{1}{2} \partial_{a} \phi^{\alpha} \partial^{a} \phi^{\alpha}-\kappa f\left(\phi^{\alpha}\right)^{2} \\
& =\frac{1}{2}\left[\left(\dot{\phi}^{\alpha}\right)\left(\dot{\phi}^{\alpha}\right)-\left(\vec{\nabla} \phi^{\alpha}\right) \cdot\left(\vec{\nabla} \phi^{\alpha}\right)\right]-\kappa f\left(\phi^{\alpha}\right)^{2},
\end{aligned}
$$

where $\kappa$ is a proportionality constant; the LM model for this field will be 


$$
\begin{aligned}
\mathcal{L} & =-\frac{1}{2} \partial_{a} \phi^{\alpha} \partial^{a} \phi^{\alpha}-\lambda f\left(\phi^{\alpha}\right) \\
& =\frac{1}{2}\left[\left(\dot{\phi}^{\alpha}\right)\left(\dot{\phi}^{\alpha}\right)-\left(\vec{\nabla} \phi^{\alpha}\right) \cdot\left(\vec{\nabla} \phi^{\alpha}\right)\right]-\lambda f\left(\phi^{\alpha}\right),
\end{aligned}
$$

where $\lambda$ is a Lagrange multiplier field. In both cases, we have chosen a time coordinate $t$ and performed a $3+1$ decomposition of the tensors; a dot over a quantity (e.g., $\dot{\phi}^{\alpha}$ ) will denote its derivative with respect to this time coordinate, while spatial derivatives will be denoted with either $\vec{\nabla}$ or $\partial_{i}$ depending on the expression.

\section{A. Potential model}

The d.o.f. for the model (5) are particularly easy to count. The momenta conjugate to the fields are all well-defined,

$$
\pi^{\alpha}=\frac{\partial \mathcal{L}}{\partial \dot{\phi}^{\alpha}}=\dot{\phi}^{\alpha}
$$

The Hamiltonian density is therefore

$$
\mathcal{H}_{0}=\pi^{\alpha} \dot{\phi}^{\alpha}-\mathcal{L}=\frac{1}{2}\left[\pi^{\alpha} \pi^{\alpha}+\left(\vec{\nabla} \phi^{\alpha}\right) \cdot\left(\vec{\nabla} \phi^{\alpha}\right)\right]+\kappa f\left(\phi^{\alpha}\right)^{2} .
$$

Nothing further is required here; we have no primary constraints on the initial data, and the Hamiltonian obtained by integrating $\mathcal{H}_{0}$ over space will generate the field dynamics. We thus have the $N$ d.o.f. one would expect.

\section{B. Lagrange-multiplier model}

Counting the d.o.f. for the model (6) requires a bit more effort. As the kinetic term of (6) is the same as that of (5), the momenta conjugate to the scalars $\phi^{\alpha}$ are again defined by (7). The difficulty arises due to the Lagrange multiplier $\lambda$. From the perspective of the model, it is just another field, but its associated momentum vanishes automatically,

$$
\varpi=\frac{\partial \mathcal{L}}{\partial \dot{\lambda}}=0 \equiv \Phi
$$

We thus have a primary constraint, $\Phi=0$, on this theory. The "base Hamiltonian density" $\mathcal{H}_{0}=\pi^{\alpha} \dot{\phi}^{\alpha}-\mathcal{L}$ must then be modified to obtain the "augmented Hamiltonian density" $\mathcal{H}_{A}$ by adding this primary constraint multiplied by an auxiliary Lagrange multiplier $u_{\lambda}{ }^{1}$

\footnotetext{
${ }^{1}$ Here and throughout, we will need to distinguish between the "real" Lagrange multiplier that appears in the original Lagrangian and the "auxiliary" Lagrange multipliers that are used to construct a Hamiltonian for the model. In general, we will only have one real Lagrange multiplier at a time, which we will denote with $\lambda$; auxiliary Lagrange multipliers will be denoted with the symbol $u$, possibly with subscripts or diacritical marks.
}

$\mathcal{H}_{A}=\mathcal{H}_{0}+u_{\lambda} \varpi=\frac{1}{2}\left[\left(\pi^{\alpha}\right)^{2}+\left(\vec{\nabla} \phi^{\alpha}\right)^{2}\right]+\lambda f\left(\phi^{\alpha}\right)+u_{\lambda} \varpi$.

We now need to ensure that this constraint is preserved by the time-evolution of the system; in other words, we must have $\dot{\varpi}=\left\{\varpi, H_{A}\right\}=0$, where $H_{A} \equiv \int \mathcal{H}_{A} d^{3} x{ }^{2}$ If this Poisson bracket does not vanish identically, this demand will yield a secondary constraint $\Psi_{1}=0$. The demand that this constraint be preserved may lead to new secondary constraints $\Psi_{2}=0, \Psi_{3}=0$, and so forth, which must themselves be conserved. We will refer to the stage at which a secondary constraint arises as its "order". In other words, if $\Psi_{1}$ ensures the preservation of a primary constraint, it is a "first-order secondary constraint"; if $\Psi_{2}$ ensures the preservation of $\Psi_{1}$, it is a "second-order secondary constraint"; and so on. In this process, it may occur that the preservation of these constraints allows us to determine the auxiliary Lagrange multiplier $u_{\lambda}$ introduced above. The process is continued until all constraints are known to be automatically conserved or a contradiction is reached.

With this in mind, we derive the secondary constraints for this model. We first have

$$
0=\dot{\varpi}=\left\{\varpi, H_{A}\right\}=-\frac{\delta H_{A}}{\delta \lambda}=f\left(\phi^{\alpha}\right) .
$$

Thus, $\Psi_{1}=f\left(\phi^{\alpha}\right)=0$ is a secondary constraint. We repeat this procedure, obtaining another secondary constraint,

$\dot{\Psi}_{1}=\left\{\Psi_{1}, H_{A}\right\}=\frac{\partial f\left(\phi^{\alpha}\right)}{\partial \phi^{\beta}} \frac{\delta H_{A}}{\delta \pi^{\beta}}=\left(\delta_{\beta} f\right) \pi^{\beta} \equiv \Psi_{2}$,

where we have defined $\delta_{\beta} f=\delta f / \delta \phi^{\beta}$. (Higher derivatives will be defined similarly.) $\Psi_{2}$ must also be conserved, which leads to a third secondary constraint,

$$
\begin{aligned}
\dot{\Psi}_{2} & =\left\{\Psi_{2}, H_{A}\right\}=-\delta_{\alpha} f\left[-\nabla^{2} \phi^{\alpha}+\lambda \delta_{\alpha} f\right]+\pi^{\alpha} \pi^{\beta}\left(\delta_{\alpha \beta} f\right) \\
& \equiv \Psi_{3} .
\end{aligned}
$$

Finally, demanding that $\Psi_{3}$ be conserved allows us to determine the auxiliary Lagrange multiplier $u_{\lambda}$; this is because $\Psi_{3}$ is itself dependent on $\lambda$,

$$
\begin{aligned}
\dot{\Psi}_{3} & =\left\{\Psi_{3}, H_{A}\right\} \\
& =\frac{\partial \Psi_{3}}{\partial \phi^{\gamma}} \frac{\delta H_{A}}{\delta \pi^{\gamma}}-\frac{\partial \Psi_{3}}{\partial \pi^{\gamma}} \frac{\delta H_{A}}{\delta \phi^{\gamma}}+\frac{\partial \Psi_{3}}{\partial \lambda} \frac{\delta H_{A}}{\delta \varpi} .
\end{aligned}
$$

\footnotetext{
${ }^{2} \mathrm{We}$ are playing a bit fast and loose with notation here; in Hamiltonian field theory, the Poisson bracket is only rigorously defined for a functional with a single real value, not for a field which is a function of space. A more rigorous definition of what we mean by an expression like $\left\{\varpi, H_{A}\right\}$ is given in the Appendix.
} 
When the dust settles, we obtain

$$
\begin{aligned}
\dot{\Psi}_{3}= & \pi^{\gamma}\left[\left(\delta_{\alpha \gamma} f\right)\left(3 \nabla^{2} \phi^{\alpha}-4 \lambda \delta_{\alpha} f\right)+\nabla^{2}\left(\delta_{\alpha} f\right)+\pi^{\alpha} \pi^{\beta}\left(\delta_{\alpha \beta \gamma} f\right)\right] \\
& -\left(\delta_{\alpha} f\right)\left(\delta_{\alpha} f\right) u_{\lambda} .
\end{aligned}
$$

So long as $\delta_{\alpha} f \neq 0$ when $f=0$, this allows us to determine the previously unknown auxiliary Lagrange multiplier $u_{\lambda}$. Thus, the process terminates here.

Having determined the Hamiltonian and its constraints, we can count the d.o.f. We have $N+1$ fields, namely the multiplet $\phi^{\alpha}(\alpha=1, \ldots, N)$ and the Lagrange multiplier $\lambda$, one primary constraint $\Phi=\varpi=0$, and three secondary constraints, $\Psi_{1}=\Psi_{2}=\Psi_{3}=0$. The single auxiliary Lagrange multiplier is determined, which means that there are no unphysical gauge d.o.f. With $N+1$ fields and four constraints, the number of d.o.f. of the model (6) is therefore

$$
N_{\mathrm{dof}}=(N+1)-\frac{1}{2}(4)=N-1 .
$$

Thus, the Lagrange-multiplier theory (6) has one less d.o.f. than the corresponding potential theory (5). In this case, the conjecture outlined in the Introduction holds true.

\section{VECTOR FIELDS}

We now consider the case of a vector field $A_{a}$ which spontaneously breaks Lorentz symmetry. As the motivation behind these models is usually a spontaneous breaking of Lorentz symmetry, we want our Lagrange density to be a Lorentz scalar, without any prior geometry specified.

In any such Lagrange density, we can identify a set of "kinetic" terms $\mathcal{L}_{K}$ that depend on the derivatives of $A_{a}$. The most general kinetic term that we can write down which is quadratic in the field $A_{a}$ is ${ }^{3}$

$$
\mathcal{L}_{k}=c_{1} \partial_{a} A_{b} \partial^{a} A^{b}+c_{2}\left(\partial_{a} A^{a}\right)^{2}+c_{3} \partial_{a} A_{b} \partial^{b} A^{a} .
$$

However, since

$$
\left(\partial_{a} A^{a}\right)^{2}=\partial_{a} A_{b} \partial^{b} A^{a}+\partial_{a}\left[A^{a} \partial_{b} A^{b}-A^{b} \partial_{b} A^{a}\right],
$$

we can eliminate one of $c_{2}$ or $c_{3}$ via an integration by parts. We will therefore set $c_{2}=0$ in what follows. The familiar "Maxwell" kinetic term

$$
\mathcal{L}_{K}=-\frac{1}{4} F_{a b} F^{a b},
$$

with $F_{a b}=2 \partial_{[a} A_{b]}$ corresponds to $c_{1}=-c_{3}=-\frac{1}{2}$.

We can now perform the usual $3+1$ decomposition of the Lagrange density, writing $A_{0}$ for the $t$-component of $A_{a}$

\footnotetext{
${ }^{3}$ This follows the notation of [10], with the coefficient $c_{4}$ from that reference set equal to zero.
}

and $\vec{A}$ (or $A_{i}$ ) for its spatial components. The kinetic term (16) then becomes

$$
\begin{aligned}
\mathcal{L}_{K}= & \frac{1}{2} c_{13}\left(\dot{A}_{0}\right)^{2}-\frac{c_{1}}{2}\left(\vec{\nabla} A_{0}\right)^{2}-\frac{c_{1}}{2} \dot{\vec{A}}^{2}-c_{3} \dot{\vec{A}} \cdot \vec{\nabla} A_{0} \\
& +\frac{c_{1}}{2}\left(\partial_{i} A_{j}\right)\left(\partial^{i} A^{j}\right)+\frac{c_{3}}{2}\left(\partial_{i} A_{j}\right)\left(\partial^{j} A^{i}\right),
\end{aligned}
$$

where $c_{13} \equiv c_{1}+c_{3}$. The momenta conjugate to $A_{0}$ and $\vec{A}$ are then

$$
\Pi^{0}=\frac{\partial \mathcal{L}_{K}}{\partial \dot{A}_{0}}=c_{13} \dot{A}_{0}
$$

and

$$
\vec{\Pi}=\frac{\partial \mathcal{L}_{K}}{\partial \dot{\vec{A}}}=-c_{1} \dot{\vec{A}}-c_{3} \vec{\nabla} A_{0}
$$

These equations can be inverted to find the velocities $\dot{A}_{0}$ and $\dot{\vec{A}}$ so long as $c_{1}+c_{3} \neq 0$ and $c_{1} \neq 0$. If either of these expressions vanishes, (19) and (20) will instead yield constraint equations; we will have to handle these cases separately.

For the potential term, meanwhile, our desire for the Lagrangian to be a Lorentz scalar implies that the only possible form for the vacuum manifold function is one which sets the norm of $A_{a}$ to some constant $b$. For simplicity's sake, we will therefore choose $f\left(A_{a}\right)$ to be of the following form:

$$
f\left(A_{a}\right)=A^{a} A_{a}-b,
$$

where $b$ is a constant. Depending on the sign of $b$, the "vacuum" manifold will consist of timelike vectors $(b<0)$, spacelike vectors $(b>0)$, or null vectors $(b=0){ }^{4}$ Our potential model will then be

$$
\mathcal{L}_{P}=\mathcal{L}_{K}-\kappa f\left(A_{a}\right)^{2}=\mathcal{L}_{K}-\kappa\left(-A_{0}^{2}+\vec{A}^{2}-b\right)^{2},
$$

where $\kappa$ is again a proportionality constant; the LM model will be

$$
\mathcal{L}_{\mathrm{LM}}=\mathcal{L}_{K}-\lambda f\left(A_{a}\right)=\mathcal{L}_{K}-\lambda\left(-A_{0}^{2}+\vec{A}^{2}-b\right) .
$$

For compactness, I will denote the four-norm of $A_{a}$ as $A^{2}=-A_{0}^{2}+\vec{A}^{2}$; the norm of the spatial part on its own will always be denoted by $\vec{A}^{2}$.

\footnotetext{
${ }^{4}$ As spontaneous symmetry breaking implies a nonzero field value, one would normally exclude the case $b=0$ to ensure that $A_{a}=0$ is not in the vacuum manifold. However, this is not necessary for the analysis which follows.
} 


\section{A. General case: $c_{13} \neq 0, c_{1} \neq 0$}

\section{Potential model}

Performing a Legendre transform on $\mathcal{L}_{P}$ (22) to obtain the Hamiltonian density, we obtain a base Hamiltonian density of

$$
\begin{aligned}
\mathcal{H}_{B}= & \frac{1}{2 c_{13}} \Pi_{0}^{2}-\frac{1}{2 c_{1}} \vec{\Pi}^{2}+\frac{c_{1}^{2}-c_{3}^{2}}{2 c_{1}}\left(\vec{\nabla} A_{0}\right)^{2} \\
& -\frac{c_{3}}{c_{1}} \vec{\Pi} \cdot \vec{\nabla} A_{0}-\frac{c_{1}}{2}\left(\partial_{i} A_{j}\right)\left(\partial^{i} A^{j}\right) \\
& -\frac{c_{3}}{2}\left(\partial_{i} A_{j}\right)\left(\partial^{j} A^{i}\right)+\kappa\left(A^{2}-b\right)^{2} .
\end{aligned}
$$

The resulting theory has four fields $\left(A_{0}\right.$ and $\left.\vec{A}\right)$ and no constraints, so the process terminates here, and the base Hamiltonian is the complete Hamiltonian for the model. Counting the d.o.f., we find that

$$
N_{\text {dof }}=4-\frac{1}{2}(0)=4 .
$$

\section{Lagrange multiplier model}

For the Lagrange multiplier model (23), we have a primary constraint associated with $\lambda$,

$$
\varpi=\frac{\partial \mathcal{L}}{\partial \dot{\lambda}}=0 .
$$

We must therefore augment the Hamiltonian with an auxiliary Lagrange multiplier $u_{\lambda}$ to enforce this constraint,

$$
\begin{aligned}
\mathcal{H}_{A}= & \Pi^{0} A_{0}+\vec{\Pi} \cdot \vec{A}-\mathcal{L}_{\mathrm{LM}}+u_{\lambda} \varpi \\
= & \frac{1}{2 c_{13}} \Pi_{0}^{2}-\frac{1}{2 c_{1}} \vec{\Pi}^{2}+\frac{c_{1}^{2}-c_{3}^{2}}{2 c_{1}}\left(\vec{\nabla} A_{0}\right)^{2}-\frac{c_{3}}{c_{1}} \vec{\Pi} \cdot \vec{\nabla} A_{0} \\
& -\frac{c_{1}}{2}\left(\partial_{i} A_{j}\right)\left(\partial^{i} A^{j}\right)-\frac{c_{3}}{2}\left(\partial_{i} A_{j}\right)\left(\partial^{j} A^{i}\right) \\
& +\lambda\left(A^{2}-b\right)+u_{\lambda} \varpi .
\end{aligned}
$$

We now find the secondary constraints required for the primary constraint to be conserved under time-evolution. Taking the Poisson brackets of each constraint with the Hamiltonian in turn, we obtain three secondary constraints,

$$
\begin{gathered}
\dot{\varpi}=\left\{\varpi, H_{A}\right\}=A_{0}^{2}-\vec{A}^{2}+b \equiv \Psi_{1} ; \\
\dot{\Psi}_{1}=\left\{\Psi_{1}, H_{A}\right\} \\
=2\left[\frac{1}{c_{13}} A_{0} \Pi^{0}+\frac{1}{c_{1}} \vec{A} \cdot\left(\vec{\Pi}+c_{3} \vec{\nabla} A_{0}\right)\right] \equiv \Psi_{2} ;
\end{gathered}
$$

and

$$
\dot{\Psi}_{2}=\left\{\Psi_{2}, H_{A}\right\}=2 \lambda\left(\frac{A_{0}^{2}}{c_{13}}-\frac{\vec{A}^{2}}{c_{1}}\right)+\Xi \equiv \Psi_{3}
$$

where

$$
\begin{aligned}
\Xi \equiv & \frac{1}{c_{13}^{2}}\left(\Pi^{0}\right)^{2}-\frac{1}{c_{1}^{2}}\left(\vec{\Pi}+c_{3} \vec{\nabla} A_{0}\right)^{2} \\
& +\frac{c_{3}}{c_{1} c_{13}}\left(\vec{A} \cdot \vec{\nabla} \Pi^{0}-A_{0} \vec{\nabla} \cdot \vec{\Pi}\right)+\frac{c_{1}-c_{3}}{c_{1}} A_{0} \nabla^{2} A_{0} \\
& -\frac{c_{3}}{c_{1}} \vec{A} \cdot \vec{\nabla}(\vec{\nabla} \cdot \vec{A})+\vec{A} \cdot \nabla^{2} \vec{A}
\end{aligned}
$$

All three of the quantities $\Psi_{1}, \Psi_{2}$, and $\Psi_{3}$ must vanish for the model to be consistent.

When we take the Poisson bracket of $\Psi_{3}$ with $H_{A}$, we will obtain

$$
\begin{aligned}
\dot{\Psi}_{3}= & \left\{\Xi, H_{A}\right\}+\left\{2 \lambda\left(\frac{A_{0}^{2}}{c_{13}}-\frac{\vec{A}^{2}}{c_{1}}\right), H_{A}\right\} \\
= & \left\{\Xi, H_{A}\right\}+2 u_{\lambda}\left(\frac{A_{0}^{2}}{c_{13}}-\frac{\vec{A}^{2}}{c_{1}}\right) \\
& +4 \lambda\left[\frac{1}{c_{13}^{2}} A_{0} \Pi^{0}+\frac{1}{c_{1}^{2}} \vec{A} \cdot\left(\vec{\Pi}+c_{3} \vec{\nabla} A_{0}\right)\right] .
\end{aligned}
$$

This means that for generic initial data, for which

$$
\frac{A_{0}^{2}}{c_{13}} \neq \frac{\vec{A}^{2}}{c_{1}}
$$

we can solve (32) for $u_{\lambda}$. ${ }^{5}$ Thus, the auxiliary Lagrange multiplier $u_{\lambda}$ is determined via the self-consistency of the theory. We have five fields $\left(A_{0}, \vec{A}\right.$, and $\left.\lambda\right)$, and selfconsistency generates four constraints (one primary, three secondary), and so the total number of d.o.f. of this model is

$$
N_{\text {dof }}=5-\frac{1}{2}(4)=3
$$

Again, as expected from the conjecture, we have lost 1 d.o.f. to the Lagrange multiplier.

A related analysis was performed by Garfinkle et al. [19] in the case of Einstein-aether theory. In such models, the vector field is constrained to satisfy $A_{a} A^{a}=b=-1$; i.e., the vector field is unit and timelike. In that work, the time component $A_{0}$ of the vector field was explicitly eliminated from the Lagrangian after performing a $3+1$ decomposition, leaving the components of $\vec{A}$ as the three dynamical

\footnotetext{
${ }^{5}$ The full expression for $\left\{\Xi, H_{A}\right\}$ is complicated and not terribly illuminating, so we will not present it here. However, from the form of $\Xi$ and $\mathcal{H}_{A}$, we can see that it will depend on $A_{0}$, $\vec{A}, \Pi^{0}, \vec{\Pi}$, and $\lambda$-but it will be independent of both $\varpi$ and (more importantly) $u_{\lambda}$.
} 
fields. It was found in that case that the model did not contain any extra constraints on these three dynamical fields, if (assuming $c_{2}=c_{4}=0$, as we have done here)

$$
c_{1} \neq 0, \quad \frac{c_{3}}{c_{1}} \leq 0 .
$$

Such models were called "safe" by the authors of [19]; such a model would be expected to contain the 3 d.o.f. present in $\vec{A}$. Models with $c_{1}=0$ were called "endangered", in that they contained additional constraints on the initial data; such models would contain fewer than 3 d.o.f. Finally, models with $c_{1} \neq 0$ and $c_{3} / c_{1}>0$ were called "conditionally endangered", since the constraint structure of the equations differed at various points in configuration space.

To connect this to the present work, we note that (33) is equivalent to

$$
c_{1} A_{0}^{2}-c_{1} \vec{A}^{2}-c_{3} \vec{A}^{2} \neq 0,
$$

or, since $c_{1} \neq 0$ and $-A_{0}^{2}+\vec{A}^{2}=b$ under the constraint,

$$
b \neq-\frac{c_{3}}{c_{1}} \vec{A}^{2} .
$$

In the case where $c_{3} / c_{1} \leq 0$ and $b<0$, this is guaranteed to hold, and we therefore have no additional constraints and 3 d.o.f. However, if $c_{3} / c_{1}>0$, there can be nongeneric points in configuration space where the number of constraints changes.

\section{B. Maxwell case: $c_{13}=0, c_{1} \neq 0$}

\section{Potential model}

We now consider a vector field $A_{a}$ with a "Maxwell" kinetic term, for which $c_{1}=-c_{3}$ in (16). We again have four independent fields, namely the four components of $A_{a}$. In this case, the canonical momentum $\Pi^{0}$ defined in (19) vanishes automatically, giving us a constraint,

$$
\Pi_{0}=0 \equiv \Phi_{1} .
$$

The other three canonical momenta $\vec{\Pi}$ defined in (20) have an invertible relationship with the corresponding field velocities,

$$
\vec{\Pi}=c_{1}\left(-\dot{\vec{A}}+\vec{\nabla} A_{0}\right) .
$$

Thus, the base Hamiltonian density $\mathcal{H}_{B}$, given by

$$
\mathcal{H}_{B}=\Pi_{0} \dot{A}_{0}+\vec{\Pi} \cdot \dot{\vec{A}}-\mathcal{L}
$$

must be augmented by an auxiliary Lagrange multiplier term enforcing the constraint $\Pi^{0}=0$. After simplification, this yields

$$
\begin{aligned}
\mathcal{H}_{A}= & -\frac{1}{2 c_{1}} \vec{\Pi}^{2}+\vec{\Pi} \cdot \vec{\nabla} A_{0} \\
& -\frac{c_{1}}{2}\left[\left(\partial_{i} A_{j}\right)\left(\partial^{i} A^{j}\right)-\left(\partial_{i} A_{j}\right)\left(\partial^{j} A^{i}\right)\right] \\
& +\kappa\left(A^{2}-b\right)^{2}+u_{0} \Pi^{0} .
\end{aligned}
$$

Once again, we must ensure that the primary constraint $\Phi=\Pi_{0}=0$ is conserved by the equations of motion; this again produces a series of secondary constraints,

$$
\dot{\Pi}^{0}=\left\{\Pi_{0}, H_{A}\right\}=\vec{\nabla} \cdot \vec{\Pi}+4 \kappa\left(A^{2}-b\right) A_{0} \equiv \Psi_{1}
$$

$$
\begin{aligned}
\dot{\Psi}_{1}= & \left\{\Psi_{1}, H_{A}\right\} \\
= & -8 \kappa\left[\vec{\nabla} \cdot\left(\left(A^{2}-b\right) \vec{A}\right)-\left(-3 A_{0}^{2}+\vec{A}^{2}-b\right) u_{0}\right. \\
& \left.+A_{0} \vec{A} \cdot\left(\frac{1}{c_{1}} \vec{\Pi}-\vec{\nabla} A_{0}\right)\right] .
\end{aligned}
$$

So long as $-3 A_{0}^{2}+\vec{A}^{2}-b \neq 0$, the demand that the secondary constraint $\Psi_{1}$ be preserved by the evolution determines the auxiliary Lagrange multiplier $u_{0}$ uniquely. We therefore have four fields, two constraints (one primary, one secondary), and no undetermined Lagrange multipliers; counting the d.o.f. therefore yields

$$
N_{\text {dof }}=4-\frac{1}{2}(2)=3 \text {. }
$$

\section{Lagrange multiplier model}

We now apply the same process to the vector model with a Lagrange multiplier, (23). With the addition of the Lagrange multiplier $\lambda$, we must also introduce a conjugate momentum $\varpi$. As in the scalar LM model, this vanishes identically, yielding a second primary constraint,

$$
\varpi=\frac{\partial \mathcal{L}}{\partial \dot{\lambda}}=0 \equiv \Phi_{2} .
$$

Including this constraint with an auxiliary Lagrange multiplier $u_{\lambda}$ in the Hamiltonian density gives us the augmented Hamiltonian density for the model,

$$
\begin{aligned}
\mathcal{H}_{A}= & -\frac{1}{2 c_{1}} \vec{\Pi}^{2}+\vec{\Pi} \cdot \vec{\nabla} A_{0} \\
& -\frac{c_{1}}{2}\left[\left(\partial_{i} A_{j}\right)\left(\partial^{i} A^{j}\right)-\left(\partial_{i} A_{j}\right)\left(\partial^{j} A^{i}\right)\right] \\
& +\lambda\left(A^{2}-b\right)+u_{0} \Pi^{0}+u_{\lambda} \varpi .
\end{aligned}
$$

We now derive the secondary constraints and see if their time-evolution fixes the auxiliary Lagrange multipliers $u_{0}$ and $u_{\lambda}$, 


$$
\begin{gathered}
\dot{\Phi}_{1}=\left\{\Pi_{0}, H_{A}\right\}=\vec{\nabla} \cdot \vec{\Pi}+2 \lambda A_{0} \equiv \Psi_{1} \\
\dot{\Phi}_{2}=\left\{\varpi, H_{A}\right\}=-A^{2}+b \equiv \Psi_{2} \\
\dot{\Psi}_{1}=\left\{\Psi_{1}, H_{A}\right\}=-\vec{\nabla} \cdot(\lambda \vec{A})+2 A_{0} u_{\lambda}+2 \lambda u_{0} \\
\dot{\Psi}_{2}=\left\{\Psi_{2}, H_{A}\right\}=2 A_{0} u_{0}-2 \vec{A} \cdot\left(\vec{\Pi}+\vec{\nabla} A_{0}\right) .
\end{gathered}
$$

Assuming $A_{0} \neq 0$, the requirement that both (48) and (49) vanish determines the auxiliary Lagrange multipliers $u_{\lambda}$ and $u_{0}$. The d.o.f. counting is therefore five fields (four components of $A_{a}$, plus $\lambda$ ), four constraints (two primary, two secondary), and no undetermined auxiliary Lagrange multipliers, for a result of

$$
N_{\text {dof }}=5-\frac{1}{2}(4)=3 .
$$

This is a surprising result: the number of d.o.f. of the theory when the vector field is "constrained" to a vacuum manifold determined by $f\left(A_{a}\right)=0$ is exactly the same as when it is "allowed" to leave this vacuum manifold. In other words, the Lagrange-multiplier "constraint" does not actually reduce the d.o.f. of the model.

We can again connect this model to the terminology of [19], as we did for the "general" LM case in Sec. IV A 2. In that work, for a model of a timelike vector field with $c_{1} \neq 0$ and $c_{13}=0$ (i.e., $c_{3} / c_{1}=-1$ ), the number of constraints was found to be zero for all points in configuration space; such a model was therefore "safe", with 3 d.o.f. at all points in field space. This is in agreement with our work here: so long as the vector $A_{a}$ is constrained to be timelike $(b<0)$, we will always have $A_{0} \neq 0$, and the above analysis holds. However, the work here also implies that a version of the Einstein-aether theory with a spacelike vector field would only be "conditionally safe", since it is possible for $A_{0}$ to vanish.

\section{C. $V$-field case: $c_{13} \neq 0, c_{1}=0$}

\section{Potential model}

In this case, we have a Lagrange density with $c_{1}=0$ and $c_{3} \neq 0$; such a field is called a " $V$-field" by Isenberg and Nester [18]. When we calculate the conjugate momenta in this case, (19) allows us to solve for the velocity $\dot{A}_{0}=$ $\Pi^{0} / c_{3}$, but (20) becomes a set of three constraints,

$$
\vec{\Phi} \equiv \vec{\Pi}+c_{3} \vec{\nabla} A_{0}=0
$$

The augmented Hamiltonian density for the potential model (22) is then

$$
\begin{aligned}
\mathcal{H}_{A}= & \Pi^{0} A_{0}+\vec{\Pi} \cdot \dot{\vec{A}}-\mathcal{L}_{P}+\vec{u} \cdot\left(\vec{\Pi}+c_{3} \vec{\nabla} A_{0}\right) \\
= & \frac{1}{2 c_{3}}\left(\Pi^{0}\right)^{2}-\frac{c_{3}}{2}\left(\partial_{i} A_{j}\right)\left(\partial^{j} A_{i}\right) \\
& +\kappa\left(A^{2}-b\right)^{2}+\vec{u} \cdot\left(\vec{\Pi}+c_{3} \vec{\nabla} A_{0}\right),
\end{aligned}
$$

where $\vec{u}$ is a vector of auxiliary Lagrange multipliers enforcing the primary constraints (50). Enforcing these primary constraints under time evolution then yields a set of three secondary constraints $\vec{\Psi}$,

$$
\dot{\vec{\Phi}}=\left\{\vec{\Phi}, H_{A}\right\}=\vec{\nabla} \Pi^{0}-c_{3} \vec{\nabla}(\vec{\nabla} \cdot \vec{A})-4 \kappa\left(A^{2}-b\right) \vec{A} \equiv \vec{\Psi} .
$$

The time-evolution of these secondary constraints, written out in terms of spatial components, is then

$$
\begin{aligned}
\dot{\Psi}_{i}= & \left\{\Psi_{i}, H_{A}\right\}=\mathcal{M}_{i j} u_{j}-4 \kappa \partial_{i}\left(\left(A^{2}-b\right) A_{0}\right) \\
& +\frac{8 \kappa}{c_{3}} A_{0} A_{i}\left(\Pi^{0}+\vec{\nabla} \cdot \vec{A}\right),
\end{aligned}
$$

where

$$
\mathcal{M}_{i j} \equiv 4 \kappa\left[\delta_{i j}\left(A^{2}-b\right)+2 A_{i} A_{j}\right] .
$$

We require that $\dot{\Psi}_{i}=0$. This can be guaranteed in Eq. (53) via an appropriate choice of $u_{j}$ so long as the matrix $\mathcal{M}_{i j}$ is invertible. For general field values, this inverse can be calculated to be

$$
\left(\mathcal{M}^{-1}\right)_{i j}=\frac{1}{4 \kappa\left(A^{2}-b\right)}\left[\delta_{i j}-\frac{2 A_{i} A_{j}}{1+2 \vec{A}^{2}}\right]
$$

and so we can solve the equation $\dot{\vec{\Psi}}=0$ for $\vec{u}$ so long as $A^{2}-b \neq 0 .{ }^{6}$ The generic theory therefore has four fields, six constraints (three primary, three secondary) and

$$
N_{\text {dof }}=4-\frac{1}{2}(6)=1
$$

degree of freedom.

\section{Lagrange multiplier model}

As in Sec. IV B 2, the switch from a potential $V$-field model to a Lagrange-multiplier $V$-field model does not actually "kill off" any d.o.f. The augmented Hamiltonian density now contains one more auxiliary Lagrange multiplier $u_{\lambda}$, which (as before) enforces the constraint $\varpi=0$,

\footnotetext{
${ }^{6}$ It is notable that this set of field values is precisely the vacuum manifold. This property becomes more important in the context of tensor models involving potentials and Lagrange multipliers and will be discussed more extensively in an upcoming work [16].
} 


$$
\begin{aligned}
\mathcal{H}_{A}= & \frac{1}{2 c_{3}}\left(\Pi^{0}\right)^{2}-\frac{c_{3}}{2}\left(\partial_{i} A_{j}\right)\left(\partial^{j} A_{i}\right)+\lambda\left(A^{2}-b\right) \\
& +\vec{u} \cdot\left(\vec{\Pi}+c_{3} \vec{\nabla} A_{0}\right)+u_{\lambda} \varpi .
\end{aligned}
$$

We thus have four primary constraints, $\vec{\Phi}=0$ [as defined in (50)] and $\varpi=0$. Requiring that $\dot{\vec{\Phi}}=0$ and $\dot{\varpi}=0$ then yields four secondary constraints, which I will denote by $\vec{\Psi}$ and $\Psi$,

$$
\begin{aligned}
\dot{\vec{\Phi}}=\left\{\vec{\Phi}, H_{A}\right\} & =\vec{\nabla} \Pi^{0}-c_{3} \vec{\nabla}(\vec{\nabla} \cdot \vec{A})-2 \lambda \vec{A} \equiv \vec{\Psi}, \\
\dot{\varpi} & =\left\{\varpi, H_{A}\right\}=A^{2}-b \equiv \Psi .
\end{aligned}
$$

The time-evolution of these secondary constraints is then

$$
\begin{gathered}
\dot{\vec{\Psi}}=\left\{\vec{\Psi}, H_{A}\right\}=2\left[\vec{\nabla}\left(\lambda A_{0}\right)-u_{\lambda} \vec{A}+\lambda \vec{u}\right] \\
\dot{\Psi}=\left\{\Psi, H_{A}\right\}=\vec{A} \cdot \vec{u}-\frac{1}{c_{3}} A_{0} \Pi^{0} .
\end{gathered}
$$

These equations determine all four auxiliary Lagrange multipliers $\vec{u}$ and $u_{\lambda}$ so long as $\vec{A} \neq 0$ and $\lambda \neq 0$; in this case, we have

$$
u_{\lambda}=\frac{1}{\vec{A}^{2}}\left[\vec{A} \cdot \vec{\nabla}\left(\lambda A_{0}\right)+\frac{1}{c_{3}} \lambda A_{0} \Pi^{0}\right]
$$

and

$$
\vec{u}=\frac{1}{\lambda}\left[u_{\lambda} \vec{A}-\vec{\nabla}\left(\lambda A_{0}\right)\right]
$$

Thus, for generic initial data, we are done. We have five fields, four primary constraints, and four secondary constraints, and so the number of d.o.f. is

$$
N_{\text {dof }}=5-\frac{1}{2}(8)=1 \text {. }
$$

As for the Maxwellian vector theory in Sec. IV B 2, the addition of a Lagrange multiplier to a $V$-field model does not reduce its d.o.f.

This analysis is again in agreement with the work of Garfinkle et al. [19]. For a model with $c_{1}=0$, they find that the Einstein-aether theory contains additional initial data constraints on the three dynamical fields $\vec{A}$ and is therefore "endangered". In the present work, we have confirmed this result: this model does indeed contain fewer than 3 d.o.f. ${ }^{7}$

\footnotetext{
${ }^{7}$ The cases $\vec{A}=0$ and $\lambda=0$ were excluded from the above analysis. In this case, one would have to look at the time evolution of the quantities in (60) and (61), generate one or more secondorder secondary constraints, and attempt to solve these for the auxiliary Lagrange multipliers. In any event, this would generate a model with no more than 1 d.o.f. (if the resulting model was even consistent at such points in configuration space).
}

\section{DISCUSSION}

\section{A. Generalization}

We have found that a field theory model in flat spacetime may or may not "lose" a d.o.f. when a constraint is added to the system via a Lagrange multiplier. Specifically, scalar models (Sec. III) and general vector models (Sec. IV A) lose a d.o.f. when we replace a potential with a Lagrange multiplier; but Maxwell-type and $V$-type vector models (Secs. IV B and IV C, respectively) retain the same number of d.o.f. regardless of whether the field values are governed by a potential or by a Lagrange multiplier.

There is an obvious difference between these cases. In those models where there are no primary constraints in the potential model, a Lagrange multiplier eliminates a d.o.f. In contrast, in the models where the potential model does contain primary constraints, the field theory retains the same number of d.o.f. when a constraint is imposed via a Lagrange multiplier.

The reason for this difference can be traced to a particular feature of the models we have examined. In those models containing primary constraints, the conservation of the first-order secondary constraints leads to an equation that determines the auxiliary Lagrange multiplier $u_{\lambda}$ [Eqs. (48) and (60) for the Maxwell-like and $V$-field models, respectively]. In those models without primary constraints, $u_{\lambda}$ is only determined once we require that higher-order secondary constraints [specifically, the third-order secondary constraints in Eqs. (13) and (30)] be conserved.

To extend this to a general statement, we first note that the primary constraints for a potential model and its corresponding Lagrange multiplier model are simply related. If the primary constraints for the potential model are a set of $M$ functions $\left\{\Phi_{1}, \ldots, \Phi_{M}\right\}$, then the primary constraints for the corresponding Lagrange multiplier model will simply be $\left\{\Phi_{1}, \ldots, \Phi_{M}, \varpi\right\}$, where $\varpi$ is the conjugate momentum to the Lagrange multiplier $\lambda$. Moreover, $\varpi$ will commute with all of the primary constraints that derive from the potential model, since none of these constraints depend on $\lambda$.

The augmented Hamiltonian density will then be the base Hamiltonian density with terms added to impose the constraints,

$$
\mathcal{H}_{A}=\mathcal{H}_{0}+u_{I} \Phi_{I}+u_{\lambda} \varpi
$$

(Here and in what follows, repeated capitalized Roman indices are summed from 1 to $M$.) The first-order secondary constraint required in order to maintain $\varpi=0$ under time evolution will then be

$$
\Psi_{\lambda}=\left\{\varpi, H_{A}\right\}=-\frac{\delta H_{A}}{\delta \lambda}=f\left(\psi^{\alpha}\right),
$$

where $\psi^{\alpha}$ here stands for the collection of fields in the model. In addition, there will be a set of first-order 
secondary constraints $\Psi_{I}(I=1, \ldots, M)$, each derived from the requirement that $\dot{\Phi}_{I}=0$; these are given by

$$
\Psi_{I}=\left\{\Phi_{I}, H_{A}\right\}
$$

Now consider the time-evolution of the first-order secondary constraints. The time derivative of $\Psi_{\lambda}$ will be independent of $u_{\lambda}$, though it will generally depend on the other auxiliary Lagrange multipliers $u_{I}$,

$\dot{\Psi}_{\lambda} \supset u_{J}\left\{\Psi_{\lambda}, \Phi_{J}\right\}+u_{\lambda}\left\{\Psi_{\lambda}, \varpi\right\}=u_{J}\left\{f\left(\psi^{\alpha}\right), \Phi_{J}\right\}$.

Note that $\left\{f\left(\psi^{\alpha}\right), \varpi\right\}=0$ since $f\left(\psi^{\alpha}\right)$ is independent of $\lambda$. Meanwhile, the expression $\dot{\Psi}_{I}$ (for arbitrary $I$ ) will contain terms of the form

$$
\dot{\Psi}_{I}=\left\{\Psi_{I}, H_{A}\right\} \supset u_{J}\left\{\Psi_{I}, \Phi_{J}\right\}+u_{\lambda}\left\{\Psi_{I}, \varpi\right\} .
$$

Equations (68) and (69) together imply that if

$$
\left\{\Psi_{I}, \varpi\right\}=0,
$$

then the equations for conservation of the constraints $\left(\dot{\Psi}_{I}=0\right.$ and $\left.\dot{\Psi}_{\lambda}=0\right)$ do not contain $u_{\lambda}$, leaving this auxiliary Lagrange multiplier undetermined at this order. If this occurs, then we must proceed to find additional second- and higher-order secondary constraints. Since we have more than two additional constraints, but only one additional d.o.f. from $\lambda$ itself, we conclude that in such cases, the Lagrange-multiplier model will have fewer d.o.f. than the potential model. ${ }^{8}$

This condition (70) can be greatly elucidated via use of the Jacobi identity. Specifically, we have

$$
\left\{\left\{\Phi_{I}, H_{A}\right\}, \varpi\right\}+\left\{\left\{H_{A}, \varpi\right\}, \Phi_{I}\right\}+\left\{\left\{\varpi, \Phi_{I}\right\}, H_{A}\right\}=0
$$

for any primary constraint $\Phi_{I}$. Since $\varpi$ commutes with the rest of these primary constraints, the last term automatically vanishes; and applying (66) and (67) yields the equation

$$
\left\{\Psi_{I}, \varpi\right\}=-\left\{f\left(\psi^{\alpha}\right), \Phi_{I}\right\}
$$

Thus, the Eq. (69) will leave $u_{\lambda}$ undetermined, and the Lagrange multiplier will reduce the d.o.f. of the model, so long as

$$
\left\{f\left(\psi^{\alpha}\right), \Phi_{I}\right\}=0
$$

i.e., the vacuum manifold function $f\left(\psi^{\alpha}\right)$ commutes with all the primary constraints.

\footnotetext{
${ }^{8}$ It is also conceivable that $u_{\lambda}$ could remain undetermined even after the process of finding the constraints is completed. This would also reduce the number of d.o.f. in the final counting.
}

Note that this result implies that a model that is "already constrained" by primary constraints from its kinetic term could potentially be "further constrained" by the introduction of a Lagrange multiplier. This did not occur in any of the vector models from Sec. IV, as all of those models containing primary constraints also had $\left\{f\left(\psi^{\alpha}\right), \Phi_{I}\right\} \neq 0$. Examples of "already-constrained" models in which a Lagrange multiplier further reduces the model's d.o.f. can be found in the next section.

\section{B. Lagrange-multiplier models in dynamical spacetimes}

The number of d.o.f. of a field theory in flat spacetime is not always simply related to the number of d.o.f. it possesses in a curved, dynamical spacetime. It is wellknown that diffeomorphism-invariant field theories have primary constraints corresponding to the nondynamical nature of the lapse and shift functions; when we pass to a dynamical spacetime, we both introduce new fields (the ten metric components) as well as new constraints. ${ }^{9}$ Perhaps less well-known, but equally important, is that d.o.f. which are unphysical (gauge or constraint) in flat spacetime can become "activated" in a minimally coupled curvedspacetime theory [18]. This occurs due to the fact that the covariant derivative of a tensor field (unlike that of a scalar) depends on the derivatives of the metric. The "minimally coupled" kinetic term for a tensor field therefore contains couplings between the metric derivatives and the tensor field derivatives, which can turn equations that were constraints or gauge d.o.f. in flat spacetime into dynamical equations in curved spacetime, and vice versa.

In light of these facts, we might then ask how much of the above analysis would carry over to dynamical spacetimes. Given the critical role played by the constraints in this analysis, it is natural to ask whether a Lagrangemultiplier model in a dynamical curved spacetime would lose any d.o.f. relative to the corresponding potential model in a dynamical curved spacetime.

The condition (73) sheds some light on this question. We know that if $u_{\lambda}$ remains undetermined when we require conservation of the first-order secondary constraints, then we will in general have to find higher-order secondary constraints, leading to a reduction of the d.o.f. of the theory relative to the corresponding potential model. This will occur when the vacuum manifold function $f\left(\psi^{\alpha}\right)$ commutes with the primary constraints of the theory.

Any diffeomorphism-invariant theory, when decomposed into $3+1$ form, will contain terms involving the lapse $N$ and shift $N^{a}$; these are related to the spacetime metric $g^{a b}$ and the induced spatial metric $h^{a b}$ by

$$
g^{a b}=h^{a b}-\frac{1}{N^{2}}\left(t^{a}-N^{a}\right)\left(t^{b}-N^{b}\right),
$$

\footnotetext{
${ }^{9}$ See $[20,21]$ for a detailed description of the Hamiltonian formulation of general relativity.
} 
where $t^{a}$ is the vector field we have chosen to correspond to "time flow" in our decomposition. We can then write down the Einstein-Hilbert action in terms of this induced metric, the lapse, and the shift. As the lapse and shift can be arbitrarily specified, the are effectively "gauge quantities" corresponding to diffeomorphism invariance; thus, their time derivatives do not appear in the Lagrange density of the theory when it is decomposed. In the Dirac-Bergmann formalism, there are therefore primary constraints on the momenta conjugate to these quantities,

$$
\Pi \equiv \frac{\partial L}{\partial \dot{N}}=0, \quad \Pi_{a} \equiv \frac{\partial L}{\partial \dot{N}^{a}}=0 .
$$

The question is then whether the vacuum manifold function $f\left(\psi^{\alpha}\right)$ commutes with these primary constraints. But this is easy enough to see, since

$$
\left\{f\left(\psi^{\alpha}\right), \Pi\right\}=\frac{\delta f}{\delta N}, \quad\left\{f\left(\psi^{\alpha}\right), \Pi_{a}\right\}=\frac{\delta f}{\delta N^{a}} .
$$

Thus, the question of whether the Lagrange multiplier reduces the number of constraints is reduced to the question of whether the vacuum manifold function depends on the lapse and shift. In particular, for a collection of scalar fields in curved spacetime (the dynamical-spacetime analogue of Sec. III), the vacuum manifold function will be independent of the metric, and so there is no way for the lapse or shift functions to enter into it. We would therefore expect that a Lagrange-multiplier model containing $N$ scalars would have fewer than $N$ d.o.f. attributable to the scalars. ${ }^{10}$

However, for a function of a vector field $A_{a}$, the norm of the vector field $A_{a}$ will depend on the lapse and shift functions,

$$
\begin{aligned}
A_{a} A_{b} g^{a b} & =A_{a} h^{a b} A_{b}-\frac{\left(\left(t^{a}-N^{a}\right) A_{a}\right)^{2}}{N^{2}} \\
& =A_{a}^{\perp} h^{a b} A_{b}^{\perp}-\frac{\left(A_{t}-N^{a} A_{a}^{\perp}\right)^{2}}{N^{2}},
\end{aligned}
$$

where $A_{t}=t^{a} A_{a}$ and $A_{a}^{\perp}=h_{a}{ }^{b} A_{b}$. Any function of the spacetime norm of $A_{a}$ will therefore depend on the lapse and shift, and so the vacuum manifold function will not commute with the primary constraints of the theory. Given the results stated above, it seems unlikely that the Lagrange multiplier would reduce the number of d.o.f. of such a theory.

It is interesting to note that this coupling occurs even if the flat-spacetime theory does not contain any primary constraints, as in the general vector models described in Sec. IVA. Since the conservation of the first-order secondary constraints determines the auxiliary multiplier $u_{\lambda}$ in this case, rather than giving rise to further constraints,

\footnotetext{
${ }^{10}$ As there is no coupling between the kinetic terms of the scalar and the metric, it seems likely that there would also still be 2 d.o.f. attributable to the metric itself.
}

one would conclude that the number of d.o.f. of a general vector theory in curved spacetime would not be reduced by the presence of a Lagrange multiplier, in contrast to the situation in flat spacetime. In fact, this is confirmed by known results. A model consisting of a vector field in a curved spacetime with a "generic" kinetic term (as in Sec. IV A) will contain 2 "metric" d.o.f. and three "vector" d.o.f., regardless of whether the vector is forced to a nonzero expectation value by a Lagrange multiplier [22] or by a potential $[18,23]$.

\section{Potential models in the low-energy limit}

In classical particle mechanics, it is common to think of a constrained system in relation to an unconstrained system with a potential energy. In the limit where the potential energy becomes infinitely strong, it can be shown that the dynamics of the unconstrained system reduce to those of a system constrained to lie only in the minimum of the potential [24]. It is therefore common, in the analysis of constrained systems, to simply include one or more Lagrange multipliers that enforce the constraints. In general, each Lagrange multiplier reduces the number of d.o.f. of the system by one.

One might think that this general picture could be carried over to field theory. In particular, a set of fields in a potential could be thought of as possessing a certain number of massive modes (corresponding to oscillations in field-space directions in which the potential increases) and a certain number of massless modes (oscillations in field-space directions in which the potential is flat). One could then construct a low-energy effective field theory in which the massive modes have "frozen out", reducing the number of d.o.f. of the model. In this low-energy limit, one would expect the fields to always lie in their vacuum manifold, effectively being constrained there. Hence, one would think that the Lagrange-multiplier version of a potential theory would nicely correspond to the low-energy behavior of the corresponding potential theory.

The results of this work, however, show that the picture is not so simple. While this simple picture holds for scalar fields in flat spacetime, it seems quite unlikely that the lowenergy limit of a Maxwell-type or $V$-type vector field in a potential would correspond to a model with the same kinetic term but containing a Lagrange multiplier. One would expect the low-energy limit to have fewer d.o.f. than the full potential model, but in these cases, the Lagrangemultiplier models and the corresponding potential models have the same number of d.o.f. While the low-energy limit of some such models has been investigated $[23,25,26]$, the Lagrange-multiplier models would necessarily have a different behavior.

In fact, this feature was noted in [23] in the context of a vector field with a "Maxwell" kinetic term. In Sec. IV C of that work, it was noted that the Lagrange-multiplier model only corresponded to the low-energy ("infinite-mass") limit 
of the potential model if the Lagrange multiplier $\lambda$ was set to zero by fiat. However, for a generic solution $\lambda$ will not vanish; the vanishing of the secondary constraint in Eq. (46) requires that $\lambda=-\vec{\nabla} \cdot \vec{\Pi} / 2 A_{0}$. In other words, one must restrict the class of solutions under consideration-i.e., further reduce the number of d.o.f.- to obtain the lowenergy limit of a potential model from the corresponding Lagrange-multiplier model. This work shows that this lack of direct correspondence is a common feature of models in which tensor fields take on a vacuum expectation value.

\section{ACKNOWLEDGMENTS}

I would like to thank D. Garfinkle, T. Jacobson, and J. Tasson for helpful discussion and correspondence on this subject. This research was supported in part by Perimeter Institute for Theoretical Physics. Research at Perimeter Institute is supported by the Government of Canada through Industry Canada and by the Province of Ontario through the Ministry of Economic Development \& Innovation.

\section{APPENDIX: POISSON BRACKETS AND FUNCTIONALS}

In calculating the time-evolution of a field quantity in Hamiltonian field theory, one would like to take the Poisson bracket of a field $\psi^{\alpha}(x)$ with the Hamiltonian $H$ to find the time-evolution of the field at $x$,

$$
\dot{\psi}^{\alpha}(x)=\left\{\psi^{\alpha}(x), H\right\} .
$$

However, one does have to be careful with this notation, as the Poisson bracket is only rigorously defined on realvalued field functionals, not on functions of space like $\psi^{\alpha}$. Specifically, we have

$\left\{G_{1}, G_{2}\right\} \equiv \int d^{3} z\left[\frac{\delta G_{1}}{\delta \psi^{\alpha}(z)} \frac{\delta G_{2}}{\delta \pi^{\alpha}(z)}-\frac{\delta G_{1}}{\delta \pi^{\alpha}(z)} \frac{\delta G_{2}}{\delta \psi^{\alpha}(z)}\right]$, where $\pi^{\alpha}$ is the conjugate field momentum to $\psi^{\alpha}$ (and a summation over $\alpha$ is implied), and the functional derivatives are implicitly defined via the relation

$$
\delta G=\int d^{3} z\left(\frac{\delta G}{\delta \psi^{\alpha}(z)}\right) \delta \psi^{\alpha}(z)
$$

To extend the definition (A2) of a Poisson bracket to a local field quantity $f\left(\psi^{\alpha}(x), \nabla \psi^{\alpha}(x), \ldots\right)$ constructed from field quantities at a fixed point $x$, one introduces the functional

$$
F_{x} \equiv \int d^{3} y\left[f\left(\psi^{\alpha}(y), \nabla \psi^{\alpha}(y), \ldots\right) \delta^{3}(x-y)\right]
$$

The functional derivatives in (A2) then become

$$
\begin{aligned}
\frac{\delta F_{x}}{\delta \psi^{\alpha}(z)}= & \frac{\partial f}{\partial \psi^{\alpha}} \delta^{3}(x-z)-\nabla_{a}\left[\frac{\partial f}{\partial\left(\nabla_{a} \psi^{\alpha}\right)} \delta^{3}(x-z)\right] \\
& +\ldots
\end{aligned}
$$

and similarly for $\pi^{\alpha}$, where the ellipses stand for higherorder derivatives of $\psi^{\alpha}$ (or $\pi^{\alpha}$ ), and the partial derivatives of $f$ (and their gradients) are evaluated at the point $z$. Here and throughout, I will use partial derivatives $\partial$ to denote the variation of a locally constructed field quantity with respect to one of its arguments, while the $\delta$ notation will be reserved for functional derivatives.

Under this extension, the Poisson bracket of a local field quantity $f\left(\psi^{\alpha}(x), \nabla \psi^{\alpha}(x), \ldots\right)$ with the Hamiltonian $H=$ $\int \mathcal{H} d^{3} x$ is "really" the Poisson bracket of the functional $F_{x}$ with $H$. Restricting attention to quantities that only depend on the fields $\psi^{\alpha}$ and $\pi^{\alpha}$ and their first derivatives, this Poisson bracket is

$$
\begin{aligned}
\frac{d}{d t}\left[f\left(\psi^{\alpha}, \nabla \psi^{\alpha}, \pi^{\alpha}, \nabla \pi^{\alpha}\right)\right] & =\left\{F_{x}, H\right\} \\
& =\int d^{3} z\left[\left(\frac{\partial f}{\partial \psi^{\alpha}} \delta^{3}(x-z)-\nabla_{a}\left[\frac{\partial f}{\partial\left(\nabla_{a} \psi^{\alpha}\right)} \delta^{3}(x-z)\right]\right) \frac{\delta H}{\delta \pi^{\alpha}(z)}\right. \\
& \left.\quad-\left(\frac{\partial f}{\partial \pi^{\alpha}} \delta^{3}(x-z)-\nabla_{a}\left[\frac{\partial f}{\partial\left(\nabla_{a} \pi^{\alpha}\right)} \delta^{3}(x-z)\right]\right) \frac{\delta H}{\delta \psi^{\alpha}(z)}\right] \\
& =\frac{\partial f}{\partial \psi^{\alpha}} \frac{\delta H}{\delta \pi^{\alpha}(x)}+\frac{\partial f}{\partial\left(\nabla_{a} \psi^{\alpha}\right)} \nabla_{a}\left(\frac{\delta H}{\delta \pi^{\alpha}(x)}\right)-\frac{\partial f}{\partial \pi^{\alpha}} \frac{\delta H}{\delta \psi^{\alpha}(x)}-\frac{\partial f}{\partial\left(\nabla_{a} \pi^{\alpha}\right)} \nabla_{a}\left(\frac{\delta H}{\delta \psi^{\alpha}(x)}\right),
\end{aligned}
$$

where all the field quantities are now evaluated at $x$. 
Note that this definition implies that time-evolution "commutes" with spatial derivatives when we take the Poisson bracket, as one would expect. For example, suppose that $f=\psi^{\alpha}$ and $H=\int \mathcal{H} d^{3} x$, where $\mathcal{H}$ is locally constructed from the fields. Then we have

$$
\left\{\psi^{\alpha}, H\right\}=\frac{\delta H}{\delta \pi^{\alpha}(x)}=\frac{\partial \mathcal{H}}{\partial \pi^{\alpha}},
$$

evaluated at $x$. Meanwhile, if $f=\nabla_{a} \psi^{\alpha}$, we have

$$
\begin{aligned}
\left\{\nabla_{a} \psi^{\alpha}, H\right\} & =\nabla_{a}\left(\frac{\delta H}{\delta \pi^{\alpha}(x)}\right)=\nabla_{a}\left(\frac{\partial \mathcal{H}}{\partial \pi^{\alpha}}\right) \\
& =\nabla_{a}\left(\left\{\psi^{\alpha}, H\right\}\right) .
\end{aligned}
$$

This fact simplifies the calculation of the Poisson brackets considerably.

This definition can be extended straightforwardly to quantities depending on higher derivatives of $\psi^{\alpha}$ and $\pi^{\alpha}$, and the above-mentioned commutativity extends to such cases as well. It can also be extended to the Poisson brackets of two local field quantities $f(x)$ and $g(y)$ by defining functionals $F_{x}$ and $G_{y}$ and following the same procedure. In such cases, the resulting Poisson bracket will contain a factor of $\delta^{3}(x-y)$. However, in the interests of clarity, we will elide these factors when we take the Poisson bracket of two such quantities; in other words, we will take it as understood that the first argument of such a Poisson bracket is evaluated at $x$, the second at $y$, and that the result is multiplied by $\delta^{3}(x-y)$ or its derivatives.
[1] F. Englert and R. Brout, Broken Symmetry and the Mass of Gauge Vector Mesons, Phys. Rev. Lett. 13, 321 (1964).

[2] P. W. Higgs, Broken Symmetries and the Masses of Gauge Bosons, Phys. Rev. Lett. 13, 508 (1964).

[3] G. S. Guralnik, C. R. Hagen, and T. W. B. Kibble, Global Conservation Laws and Massless Particles, Phys. Rev. Lett. 13, 585 (1964).

[4] P. W. Higgs, Spontaneous symmetry breakdown without massless bosons, Phys. Rev. 145, 1156 (1966).

[5] V. L. Ginzburg and L. D. Landau, On the theory of superconductivity, Zh. Eksp. Teor. Fiz. 20, 1064 (1950).

[6] M. Gell-Mann and M. Lévy, The axial vector current in beta decay, Nuovo Cimento 16, 705 (1960).

[7] F. Gürsey, On the symmetries of strong and weak interactions, Nuovo Cimento 16, 230 (1960).

[8] G. S. Joyce, Classical Heisenberg model, Phys. Rev. 155, 478 (1967).

[9] V. A. Kostelecký and S. Samuel, Spontaneous breaking of lorentz symmetry in string theory, Phys. Rev. D 39, 683 (1989).

[10] T. Jacobson and D. Mattingly, Gravity with a dynamical preferred frame, Phys. Rev. D 64, 024028 (2001).

[11] J. Bekenstein, Relativistic gravitation theory for the modified Newtonian dynamics paradigm, Phys. Rev. D 70, 083509 (2004).

[12] V. A. Kostelecký, Gravity, Lorentz violation, and the standard model, Phys. Rev. D 69, 105009 (2004).

[13] J. W. Moffat, Scalar-tensor-vector gravity theory, J. Cosmol. Astropart. Phys. 03 (2006) 004.
[14] B. Altschul, Q. G. Bailey, and V. A. Kostelecký, Lorentz violation with an antisymmetric tensor, Phys. Rev. D 81, 065028 (2010).

[15] Y. Nambu, Quantum electrodynamics in nonlinear gauge, Prog. Theor. Phys. Suppl. E68, 190 (1968).

[16] M. D. Seifert (in preparation).

[17] P. A. M. Dirac, Lectures on Quantum Mechanics (Yeshiva University, New York, 1964).

[18] J. Isenberg and J. Nester, The effect of gravitational interaction on classical fields: A hamilton-dirac analysis, Ann. Phys. (N.Y.) 107, 56 (1977).

[19] D. Garfinkle, J. Isenberg, and J. M. Martin-Garcia, Constraint equations in Einstein-aether theories and the weak gravitational field limit, Phys. Rev. D 86, 084009 (2012).

[20] R. M. Wald, General Relativity (University of Chicago Press, Chicago, 1984).

[21] E. Poisson, A Relativist's Toolkit: The Mathematics of BlackHole Mechanics (Cambridge University Press, Cambridge, England, 2004).

[22] T. Jacobson and D. Mattingly, Einstein-aether waves, Phys. Rev. D 70, 024003 (2004).

[23] R. Bluhm, S.-H. Fung, and V. A. Kostelecký, Spontaneous Lorentz and diffeomorphism violation, massive modes, and gravity, Phys. Rev. D 77, 065020 (2008).

[24] V. I. Arnold, Mathematical Methods of Classical Mechanics (Springer, Berlin, 1989).

[25] M.D. Seifert, Vector models of gravitational Lorentz symmetry breaking, Phys. Rev. D 79, 124012 (2009).

[26] M. D. Seifert, Generalized bumblebee models and Lorentzviolating electrodynamics, Phys. Rev. D 81, 065010 (2010). 